\title{
Clinical evaluation of physician-controlled guidewire manipulation during endoscopic ultrasound-guided hepaticogastrostomy (with video) $\square$
}

(๑) $\circledast \ominus$

\author{
Authors \\ Kyohei Nishiguchi, Takeshi Ogura, Nobu Nishioka, Saori Ueno, Atsushi Okuda, Tadahiro Yamada, Masanori Yamada, \\ Kazuya Ueshima, Kazuhide Higuchi
}

Institution

2nd Department of Internal Medicine, Osaka Medical

College, Osaka, Japan

submitted 25.8.2020

accepted after revision 19.10.2020

Bibliography

Endoscopy International Open 2021; 09: E395-E400

DOI 10.1055/a-1336-3132

ISSN 2364-3722

(C) 2021. The Author(s).

This is an open access article published by Thieme under the terms of the Creative Commons Attribution-NonDerivative-NonCommercial License, permitting copying and reproduction so long as the original work is given appropriate credit. Contents may not be used for commecial purposes, or adapted, remixed, transformed or built upon. (https://creativecommons.org/licenses/by-nc-nd/4.0/)

Georg Thieme Verlag KG, Rüdigerstraße 14,

70469 Stuttgart, Germany

Corresponding author

Takeshi Ogura, 2nd Department of Internal Medicine, Osaka Medical College, 2-7 Daigakuchou, Takatsukishi, Osaka 5698686, Japan

Fax: +81-726846532

oguratakeshi0411@yahoo.co.jp

\section{ABSTRACT}

Background and study aims Endoscopic ultrasound (EUS)-guided hepaticogastrostomy (HGS) may be most complex because of the EUS-guided biliary drainage proce- dure and variations in the course of the intrahepatic bile duct compared with the common bile duct (CBD). Appropriate guidewire insertion is essential. Physician-controlled guidewire manipulation (PCGW) might improve technical success rates of bile duct cannulation. The present study aimed to determine the technical feasibility and safety of PCGW during EUS-HGS.

Patients and methods A total of 122 consecutive patients who were scheduled to undergo EUS-HGS between October 2017 and April 2019 were prospectively registered. The primary endpoint was the technical success rate of guidewire insertion into the CBD or hepatic hilum. Guidewire insertion was considered to have failed if the HGS assistant failed to achieve manipulation.

Results The intrahepatic bile duct was successfully punctured in 120 of 122 patients. During guidewire insertion by the HGS assistant, guidewire fracture was observed in one patient. The guidewire was successfully inserted into the biliary tract and manipulated by the HGS assistant in 96 patients. PCGW was thus attempted for the remaining 23 patients. The guidewire was inserted by PCGW in all 23 patients, improving the technical success rate for guidewire insertion from $80 \%$ to $100 \%$. After tract dilation, we deployed covered metal stents and plastic stents in 117 and two patients, respectively. The overall technical success rate for EUS-HGS was 97.5\% (119/122). Adverse events comprising bile peritonitis or leakage developed in five patients.

Conclusion PCGW might contribute to improving the success rate of EUS-HGS.

\section{Introduction}

Stent deployment under endoscopic biliary drainage with endoscopic retrograde cholangiopancreatography guidance (ERCP) is an established means of treating malignant biliary obstruction [1-3]. Percutaneous transhepatic biliary drainage (PTBD) has been widely attempted in patients in whom ERCP is not possible due to duodenal obstruction or surgically altered anastomosis. However, PTBD has several disadvantages, such as the risk of self-tube removal or cosmetic issues associated with external biliary drainage.

Endoscopic ultrasound-guided biliary drainage (EUS-BD) has recently emerged [4-12]. Among EUS-BD procedures, EUSguided hepaticogastrostomy (HGS) might be the most complex, because of variations in the course of the intrahepatic bile duct compared with the common bile duct (CBD). Tract di- 
lation has been the focus of efforts toward improving technical success [13-15], but this requires appropriate guidewire insertion after the initial bile duct puncture. Liver impaction can reportedly prevent wire shearing and improve technical outcomes of guidewire insertion [16]. This technique might be challenging if the intrahepatic bile duct is not well dilated, and is thus difficult to visualize on EUS during the procedure. To overcome these disadvantages, we recently attempted EUSHGS using physician-controlled guidewire manipulation (PCGW). PCGW might improve technical success rates for bile duct cannulation under ERCP guidance [17]. Similarly, this technique might be useful to improve guidewire manipulation during EUS-HGS.

The present study aimed to determine the technical feasibility and safety of PCGW during EUS-HGS.

\section{Patients and methods}

A total of 122 patients (median age, 78 years; range, 69-88 years; 63 male) who were scheduled to undergo EUS-HGS between October 2017 and April 2019 were prospectively registered. The inclusion criteria were: failed ERCP due to inaccessible papilla using a standard ERCP scope and complication with symptomatic benign biliary stricture such as hepaticojejunostomy stricture, or advanced malignant biliary obstruction. The exclusion criteria were previous PTBD or percutaneous transhepatic gallbladder drainage. This study was approved by the Ethics Committee at Osaka Medical College (IRB No.898), and all patients provided written informed consent for all procedures associated with the study. This study protocol conformed to the ethical guidelines of the Declaration of Helsinki as reflected in a priori approval by the human research committee at the institution.

\section{Technical tips for EUS-HGS using PCGW (video)}

One experienced endoscopist (T.O.) trained in therapeutic ERCP and EUS implemented all procedures. The intrahepatic bile duct was identified under EUS guidance using GF-UCT 260 (Olympus Optical, Tokyo, Japan). The bile duct of interest was punctured using a 19G fine needle aspiration (FNA) needle (Sono Tip Pro Control; Medi-Globe GmbH, Rosenheim, Germany), and bile was aspirated. Contrast medium was injected into the biliary tract through the FNA needle, and the course of the biliary tract was evaluated ( $\triangleright$ Fig. 1a). A VisiGlide 10.025 -inch guidewire (length, 2700 mm; Olympus Medical Systems, Tokyo, Japan) was then inserted into the biliary tract and initially manipulated by one assistant endoscopist (N.N. or S.U.) who was experienced in ERCP procedures (>500 ERCP therapeutic procedures) and HGS assistant (>50 EUS-HGS procedures). If the guidewire was advanced into the periphery of the bile duct, injured the hepatic parenchyma, or became lodged between fine-needle aspiration (FNA) needles, pulling the FNA needle back into the hepatic parenchyma (liver impaction technique) might be needed to prevent adverse events (AEs) associated with guidewires, as previously reported [16]. However, during this technique, guidewire resistance is an extremely important factor for successful guidewire manipulation. If the
HGS assistant was manipulating the guidewire, feeling the delicate difference in resistance between the guidewire and echoendoscope, FNA needle, or bile duct might be difficult. In this situation, PCGW (T.O) was attempted (> Fig. 1b). During the liver impaction technique, if the guidewire was clearly identifiable on EUS images, the guidewire was manipulated under EUS guidance ( $\mathbf{F i g . 1 c}$ ). If not, the physician with the ability to feel guidewire resistance gently manipulated the guidewire under fluoroscopic guidance. After inserting the guidewire into the biliary tract, the stomach and bile duct wall were dilated using a 4-mm REN biliary dilation balloon catheter (KANE$\mathrm{KA}$, Osaka, Japan). If the stent delivery system could not be inserted into the biliary tract, the fistula was dilated using a fine 025 electrocautery dilator (Medico's HIRATA INC., Osaka, Japan). A covered, self-expandable, $10 \mathrm{~mm} \times 10-$ or $12-\mathrm{cm}$ Niti-S Biliary Covered Stent (SEMS; TaeWoong Medical, Seoul, South Korea) was deployed from the intrahepatic bile duct to the stomach using intra-scope channel release to prevent stent migration ( $\triangleright$ Fig. 1d) [18]. If the metal stent could not be inserted for any reason, an alternative Type IT plastic stent (Gadelius Medical Co., Tokyo, Japan) was deployed (\Fig. 1e). All patients underwent computed tomography 1 day after EUS-HGS to detect AEs such as bile leakage, peritonitis, or stent migration.

\section{Definition}

Mean diameter of the puncture site was measured on EUS. The primary endpoint was the technical success rate for guidewire insertion into the CBD or hepatic hilum. Guidewire insertion was considered to have failed if the HGS assistant failed to achieve guidewire manipulation. Failed guidewire manipulation by the HGS assistant was in turn defined as follows: 1) guidewire manipulation attempted for $>5$ min; 2) guidewire advanced into the periphery of the intrahepatic bile duct three times; or 3) penetration of the hepatic parenchyma by the guidewire. Bile peritonitis was diagnosed if fever, elevation of inflammatory markers in blood examination, and abdominal pain were observed within 1 day after EUS-HGS.In addition, this was diagnosed by findings of bile leak or peritonitis around the HGS stent according to computed tomography, which was performed the day after EUS-HGS. Finally, AEs were graded according to the American Society for Gastrointestinal Endoscopy [19].

\section{Results}

- Table 1 shows the characteristics of the patients $(n=88,72$ $\%$ ) with primary malignant diseases comprising pancreatic cancer $(n=41)$, cholangiocarcinoma $(n=18)$, gastric cancer $(n=$ 22 ), and others $(n=7)$. Primary benign diseases in 34 patients $(18 \%)$ comprised hepaticojejunostomy stricture $(n=22)$, as well as intrahepatic bile duct stones complicated by surgical anastomosis stricture $(n=12)$. The intrahepatic bile duct was punctured at B3 in 120 patients (98\%). Median diameter of the intrahepatic bile duct was $3.9 \mathrm{~mm}$ (range, 1.6-5.7 mm). Indications for EUS-HGS in 115 patients (94\%) comprised duodenal obstruction due to malignant tumor or surgical anastomosis. 

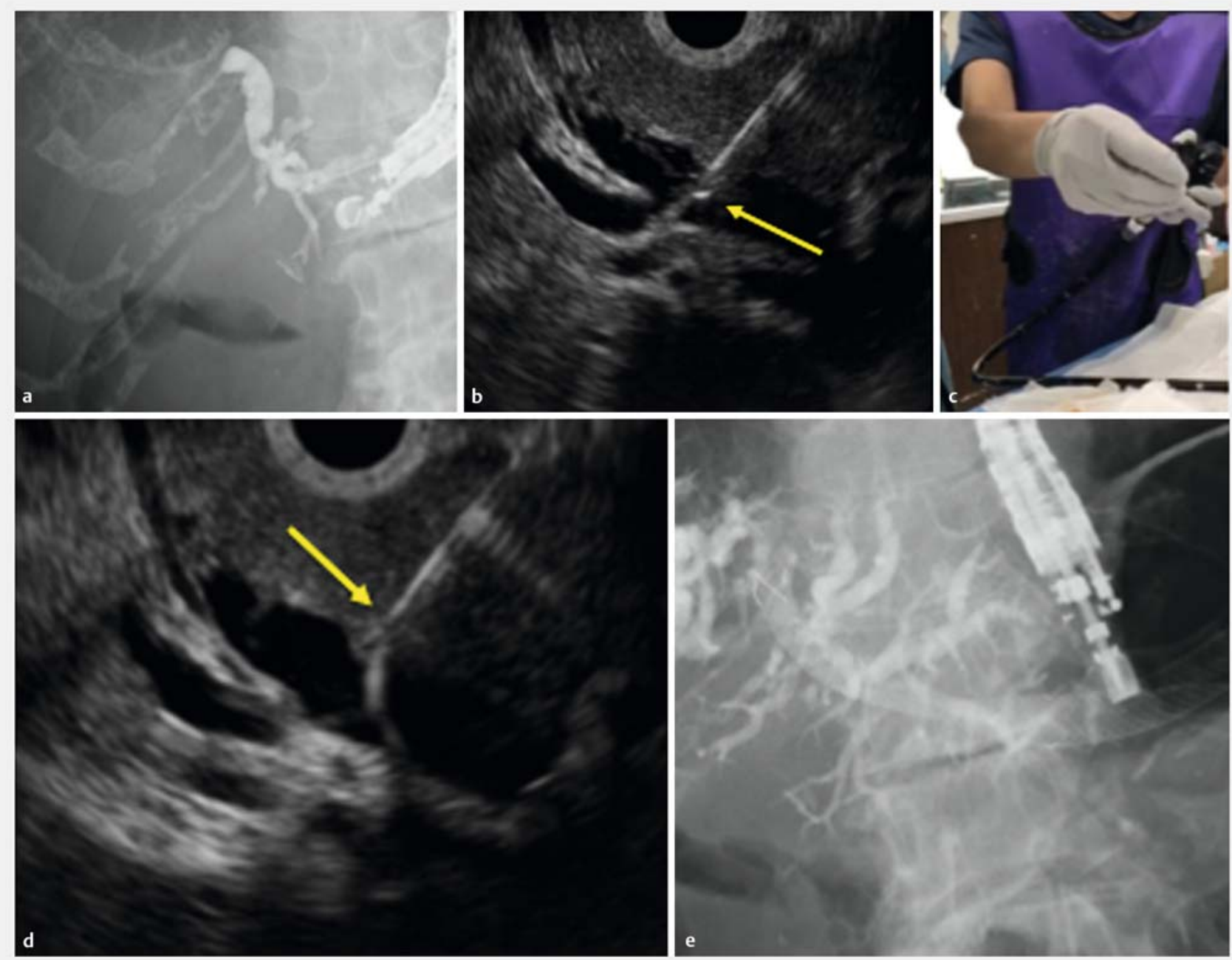

- Fig. 1 a The intrahepatic bile duct is punctured, and contrast medium is injected. b The top of the needle (arrow) is identified on EUS imaging, although the guidewire is advanced into the periphery of the bile duct. $\mathbf{c}$ Image of physician-controlled guidewire manipulation. $\mathbf{d}$ The needle itself is pulled back into the hepatic parenchyma (arrow), and the guidewire is successfully advanced into the hepatic hilum. e EUS-guided hepaticogastrostomy is successfully performed.

The flowchart in $>$ Fig. 2 shows the steps in the EUS-HGS procedure. The intrahepatic bile duct was punctured in 120 of 122 patients, but not in 2 patients with a non-dilated intrahepatic bile duct complicated with stones; they were treated surgically. These patients underwent biliary drainage under an enteroscopic approach. The guidewire was inserted into the biliary tract through the FNA needle and manipulated by the HCS assistant in 96 patients. Guidewires could not be inserted because of guidewire fracture in one patient ( $>$ Fig. 3a) who underwent PTBD. The hepatic parenchyma was injured in three patients ( $\triangleright$ Fig. $\mathbf{3 b}$ ), the guidewire became lodged in one pa-

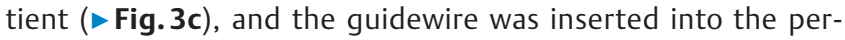
iphery of the bile duct in 19 patients ( $\triangleright$ Fig. 3d). No differences between two HGS assistants were identified. Therefore, PCGW was attempted for the 23 patients in whom the guidewire could not initially be inserted. A liver impaction technique with EUS ( Fig.4a) or fluoroscopic ( $\mathbf{F i g . 4 b )}$ guidance was attempted under PCGW ( $\mathbf{F i g . 4 c}$ ). Thereafter, a guidewire was inserted using PCGW in all 23 patients, thus improving the technical success rate for guidewire insertion from $80 \%$ to $100 \%$. The tract was dilated using a balloon catheter in 117 patients and an electrocautery dilator in 12 patients. We deployed covered SEMS and plastic stents using EUS-HGS in 117 and two patients, respectively. The overall technical success rate for EUS-HGS was $97.5 \%$ (119 of 122). Although AEs comprising bile peritonitis or leakage developed in five patients (4\%), no AEs such as guidewire sharing were seen in association with PCGW. In our study, three AEs were seen in the HGS assistant group and two were seen in the PCGW group. All AEs were successfully treated conservatively. Further, no relationship was seen between groups and AEs. 


\begin{tabular}{|c|c|}
\hline Total number of patients & 122 \\
\hline Median age (yr, range) & $78(69-88)$ \\
\hline Gender (male:female) & $63: 59$ \\
\hline \multicolumn{2}{|l|}{ Disease } \\
\hline - Malignant, n & $88(72 \%)$ \\
\hline - Pancreatic cancer & 41 \\
\hline - Cholangiocarcinoma & 18 \\
\hline - Gastric cancer & 22 \\
\hline - Other & 7 \\
\hline - Benign, $\mathrm{n}$ & $34(18 \%)$ \\
\hline - Hepaticojejunostomy stricture & 22 \\
\hline - Common bile duct stone & 8 \\
\hline - Intrahepatic bile duct stone & 4 \\
\hline \multicolumn{2}{|l|}{ Reasons for EUS-HGS } \\
\hline - Duodenal obstruction & 81 \\
\hline - Surgical anastomosis & 34 \\
\hline - Failed biliary cannulation & 7 \\
\hline \multicolumn{2}{|l|}{ Puncture site } \\
\hline - B2 & 2 \\
\hline - B3 & 120 \\
\hline Mean diameter of puncture site ( $\mathrm{mm}$, range) & $3.9(1.6-5.7)$ \\
\hline Reason for failed GW insertion, $\mathrm{n}$ & 24 \\
\hline GW insertion into the periphery of the bile duct & 19 \\
\hline GW stack & 1 \\
\hline Hepatic parenchyma injury & 3 \\
\hline GW fracture & 1 \\
\hline \multicolumn{2}{|l|}{ Adverse events } \\
\hline - Bile peritonitis & 4 \\
\hline - Bile leakage & 1 \\
\hline
\end{tabular}

\section{Discussion}

EUS-BD offers an alternative biliary drainage. Although the findings of a retrospective study with potential for patient selection bias have indicated excellent technical success rates, EUS-BD might not be that simple especially during EUS-HGS, which can be associated with AEs in the event of failure. Improved technical success rates are thus desirable.

Oh et al. compared the outcomes of 174 attempted EUSHGS procedures in 129 patients [20] using multivariate analysis. Among the 174 attempts, 54 failed and 120 were successful. Age, sex, reasons for biliary obstruction, ascites, elapsed

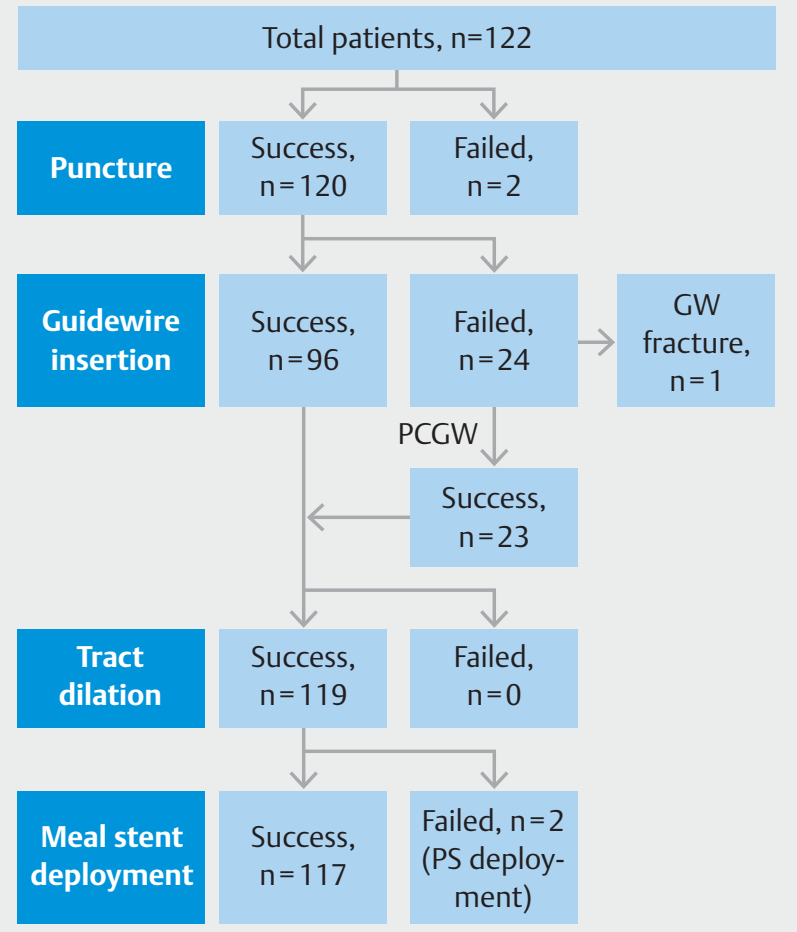

- Fig. 2 Flowchart for technical success.
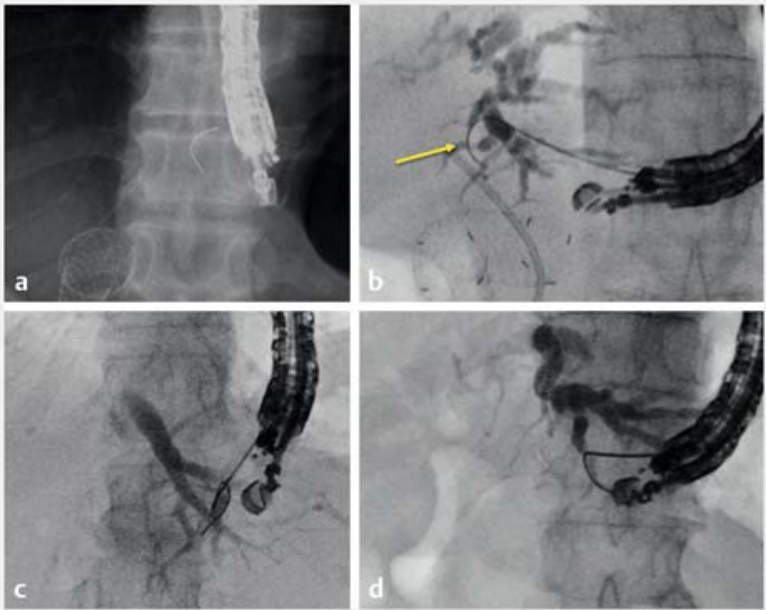

Fig. 3 a Guidewire fracture (arrow). b Liver injury due to the guidewire (arrow). c The guidewire is stacked. $\mathbf{d}$ Guidewire insertion into the periphery of the bile duct.

time, and puncture sites were not associated with technical success rates. In that study, logistic regression analysis associated low technical success rates with puncture site diameter $\leq 5 \mathrm{~mm}$ (odds ratio [OR], 3.7; 95\% confidence interval [Cl], $1.71-8.1 ; P<0.01)$ and length of the hepatic portion $>3 \mathrm{~cm}$ (OR 5.7; $95 \% \mathrm{Cl}, 2.7-12 ; P<0.01$ ). From the perspective of technical tips, guidewire manipulation and tract dilation failed in 15 (11.6\%) and 20 (15.5\%) of 129 attempts, respectively. Various 

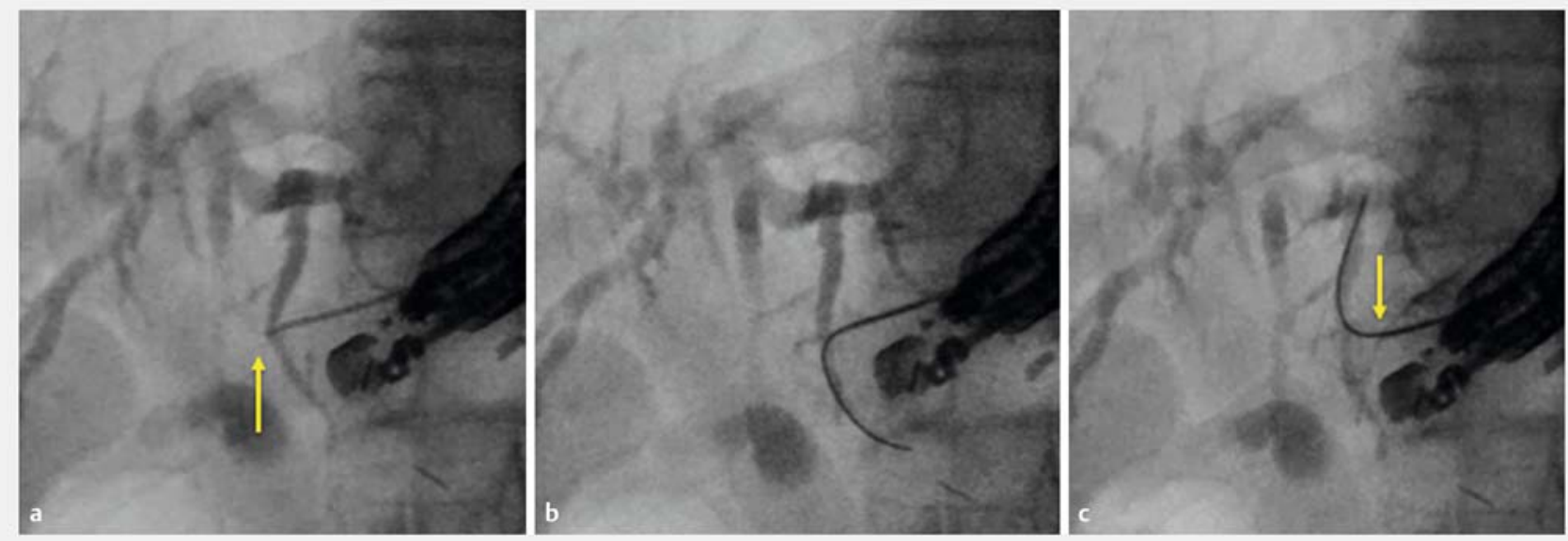

- Fig. 4 a Top of the needle (arrow). b The guidewire is advanced into the periphery of the bile duct. c The needle itself is pulled back into the hepatic parenchyma on fluoroscopic imaging (arrow), and the guidewire is successfully advanced into the hepatic parenchyma.

methods of tract dilation have been reported. Honjo et al. compared the safety and ability of ultra-tapered mechanical and electrocautery dilators for EUS-HGS and EUS-guided pancreatic duct drainage [13]. The success rate for initial tract dilation was $95.3 \%$ (61 of 64 ) in that study, and technical success rates did not differ significantly between mechanical and electrocautery dilators. However, although incidences of AEs did not differ significantly, bleeding occurred only when the tract was dilated using electrocautery. We recently described a novel dilator for EUS-HGS that might reduce the risk of bleeding caused by electrocautery [14]. Tract dilation should thus become less of an issue because of device improvement such as a fine gauge balloon catheter and mechanical or electrocautery dilator [1315].

On the other hand, the course is more complex for the intrahepatic bile duct than for the CBD, and the technical success rate for guidewire insertion should also be improved. Indeed, Vila et al. described technical evaluation of EUS-guided cholangiopancreatography for biliary and pancreatic duct drainage in non-expert hands [21]. Among 125 patients, puncture of the biliary tract or pancreatic duct was punctured under EUS guidance in 120 of 125 patients and 13 patients were injected with contrast medium. Guidewire insertion into the duct and guidewire manipulation failed in one and 18 patients, respectively. The overall technical success rate was $67.2 \%$. That study found that intraductal manipulation of the guidewire was a critically limiting step.

The present report provides technical tips for manipulating guidewires during EUS-HGS. A guidewire that is not obvious on EUS images indicates a dilated bile duct and thus, manipulation of the guidewire by the HGS assistant might prove challenging. However, guidewire resistance can be felt directly by the manipulator during PCGW. This might prevent the guidewire from becoming lodged between FNA needles. In addition, an FNA needle can be pulled only as far as the necessary distance after the guidewire is advanced into the periphery of the bile duct. Indeed, PCGW allows technical improvements. Therefore, our strategy regarding guidewire manipulation during EUS-HGS is as follows. If the guidewire is not successfully advanced by the HGS assistant, PCGW is attempted first. However, if guidewire manipulation proves unsuccessful even with PCGW, we change the puncture site. Changing to a more flexible guidewire or to a cystotome-guided guidewire technique might also be useful. However, if the guidewire is not placed in an appropriate site of the biliary tract, device insertion might be challenging. These techniques are thus not commonly attempted in our hospital. Further evaluation is needed to determine the appropriate technique after failed guidewire manipulation.

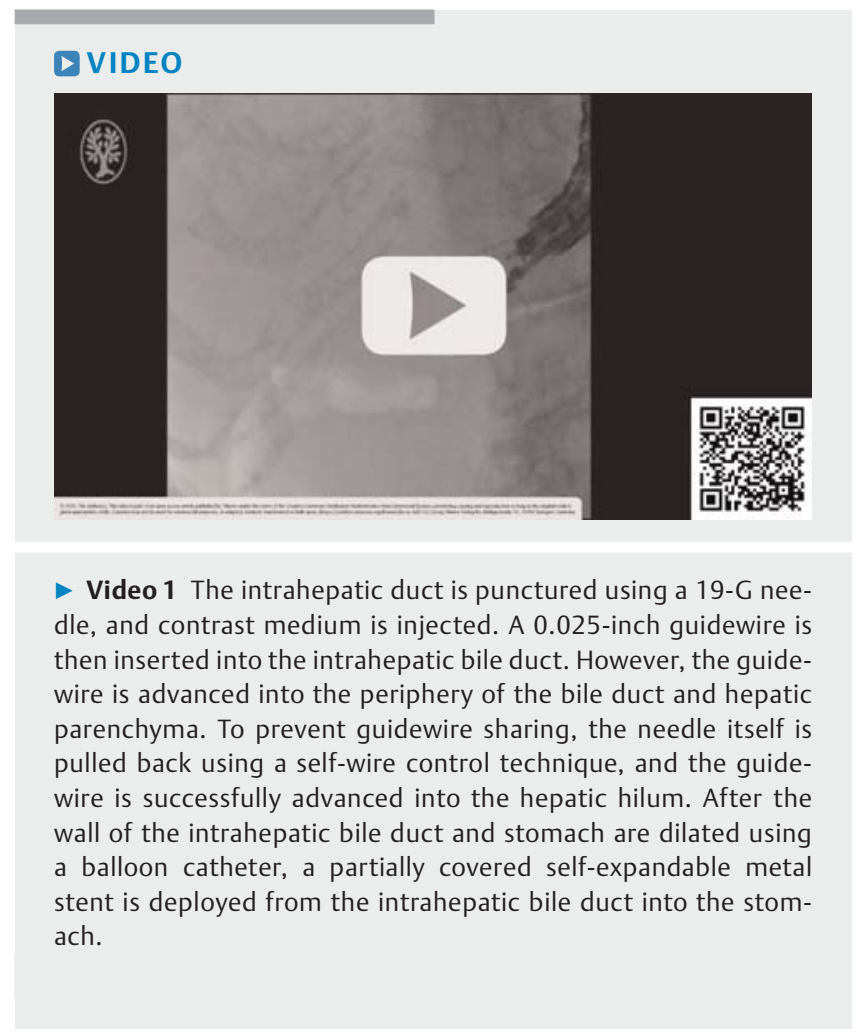


This single-arm, single-center study had several limitations, and our technique thus should be evaluated in a multicenter, prospective, randomized trial. Another limitation is that our technique requires two experienced endoscopists. This option may not be available in a majority of endoscopy units, where only one endoscopist is available per procedure. Another limitation is the increased cost of having two endoscopists per procedure. On the other hand, in many countries, expert nurses who are trained in interventional endoscopy may be able to play a role in successful EUS-HCS. Unfortunately, nurses with such expertise are not available in many Japanese hospitals. This option should also be compared with our technique in a future study.

\section{Conclusion}

In conclusion, PCGW might contribute to improving the success rate for EUS-HGS although further comparative studies are needed to verify our results.

\section{Competing interests}

The authors declare that they have no conflict of interest.

\section{References}

[1] Fogel EL, Sherman S, Park SH et al. Therapeutic biliary endoscopy. Endoscopy 2003; 35: 156-163

[2] Fujita N. Endoscopic management of biliary malignancies. Gastrointest Endosc Clin N Am 2008; 18: 565-579

[3] Gerges C, Schumacher B, Terheggen G et al. Expandable metal stents for malignant biliary obstruction. Gastrointest Endosc Clin N Am 2011; 21: 481-497

[4] Salerno R, Davies SEC, Mezzina N et al. Comprehensive review on EUSguided biliary drainage. World J Gastrointest Endosc 2019; 16: 354364

[5] Kahaleh M, Artifon ELA, Perez-Miranda M et al. EUS-guided drainage: Summary of therapeutic EUS consortium meeting. Endosc Ultrasound 2019; 8: 151-160

[6] Boulay BR, Lo SK. Endoscopic ultrasound-guided biliary drainage. Gastrointest Endosc Clin N Am 2018; 28: 171-185

[7] Teoh AYB, Dhir V, Kida M et al. Consensus guideline on the optimal management in interventional EUS procedures: results from the Asian EUS group RAND/UCLA expert panel. Gut 2018; 67: 1209-1228
[8] Baars JE, Kaffes AJ, Saxena P. EUS-guided biliary drainage: A comprehensive review of the literature. Endosc Ultrasound 2018; 7: 4-9

[9] Ogura T, Higuchi K. Technical tips for endoscopic ultrasound-guided hepaticogastrostomy. World J Gastroenterol 2016; 22: 3945-3951

[10] Hedoude A, Sportes A, Grabar S et al. Outcomes of endoscopic ultrasound-guided biliary drainage: A systematic review and meta-analysis. United European Gastroenterol J 2019; 7: 60-68

[11] Isayama H, Nakai Y, Itoi T et al. Clinical practice guideline for safe performance of endoscopic ultrasound/ultrasonography-guided biliary drainage: 2018. J Hepatobiliary Pancreat Sci 2019; 26: 249-269

[12] Dhir V, Isayama H, Itoi T et al. Endoscopic ultrasonography-guided biliary and pancreatic duct interventions. Dig Endosc 2017; 29: 472485

[13] Honjo M, Itoi T, Tsuchiya T et al. Safety and efficacy of ultra-tapered mechanical dilator for EUS-guided hepaticogastrostomy and pancreatic duct drainage compared with electrocautery dilator (with video). Endosc Ultrasound 2018; 7: 376-382

[14] Ogura T, Nakai Y, Itoi T. Novel fine gauge electrocautery dilator for endoscopic ultrasound-guided hepaticogastrostomy (with video). J Hepatobiliary Pancreat Sci 2019; 26: E3-E4

[15] Amano M, Ogura T, Onda S et al. Prospective clinical study of endoscopic ultrasound-guided biliary drainage using novel balloon catheter (with video). J Gastroenterol Hepatol 2017; 32: 716-720

[16] Ogura T, Masuda D, Takeuchi T et al. Liver impaction technique to prevent shearing of the guidewire during endoscopic ultrasoundguided hepaticogastrostomy. Endoscopy 2015; 47: E583-E584

[17] Coté GA, Ansstas M, Pawa R et al. Difficult biliary cannulation: use of physician-controlled wire-guided cannulation over a pancreatic duct stent to reduce the rate of precut sphincterotomy (with video). Gastrointest Endosc 2010; 71: 275-279

[18] Miyano A, Ogura T, Yamamoto K et al. Clinical impact of the intrascope channel stent release technique in preventing stent migration during EUS-guided hepaticogastrostomy. J Gastrointest Surg 2018; 22: 1312-1318

[19] Cotton PB, Eisen GM, Aabakken L et al. A lexicon for endoscopic adverse events: report of an ASGE workshop. Gastrointest Endosc 2010; 71: 446-454

[20] Oh D, Park DH, Song TJ et al. Optimal biliary access point and learning curve for endoscopic ultrasound-guided hepaticogastrostomy with transluminal stenting. Therap Adv Gastroenterol 2017; 10: 42-53

[21] Vila J], Pérez-Miranda M, Vazquez-Sequeiros E et al. Initial experience with EUS-guided cholangiopancreatography for biliary and pancreatic duct drainage: a Spanish national survey. Gastrointest ENdosc 2012; 76: $1133-1141$ 